\title{
Monoester Formation by Hydrolysis of Dialkyl Phthalate Migrating from Polyvinyl Chloride Products in Human Saliva
}

\author{
Tatsuhiro Niino, ${ }^{*, a}$ Tohru Ishibashi, ${ }^{a}$ Takeshi Itho, ${ }^{a}$ Senzo Sakai, ${ }^{a}$ Hazimu Ishiwata, ${ }^{b}$ \\ Takashi Yamada, ${ }^{c}$ and Sukeo Onodera ${ }^{d}$ \\ ${ }^{a}$ Tokyo Kenbikyo-in Foundation, Center of Food \& Environmental Sciences, 44-1 Nihonbashi Hakozaki-cho, Chuo-ku, Tokyo 103- \\ 0015, Japan, ${ }^{b}$ National Institute of Health Sciences, 1-18-1 Kamiyoga, Setagaya-ku, Tokyo 158-8501, Japan, 'Japan Food Additives

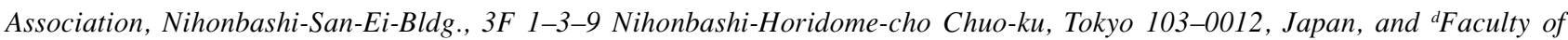 \\ Pharmaceutical Sciences, Tokyo University of Science, 12 Ichigaya-Funagawara-machi, Shinjuku-ku, Tokyo 162-0826, Japan
}

(Received February 5, 2001; Accepted February 13, 2001)

The migration dialkyl phthalate was tested in volunteers who chewed polyvinyl chloride (PVC) toy products under controlled conditions. The PVC toy samples consisted of ball A containing 100 and $185 \mathrm{mg} /$ g di-n-butyl phthalate (DBP) and di-2-ethylhexyl phthalate (DEHP) respectively, and ball $B$, containing $256 \mathrm{mg} / \mathrm{g}$ diisononyl phthalate (DINP). The migration of dialkyl phthalate into simulated saliva was also tested in vitro by shaking toy samples. The migration rates of DBP, DEHP and DINP from balls $A$ and $B$ were $11.7,44.4$ and $78.0 \mu \mathrm{g} / 10 \mathrm{~cm}^{2} / \mathrm{hr}$, respectively, in vivo, and 339,315 and $535 \mu \mathrm{g} / 10 \mathrm{~cm}^{2} / \mathrm{hr}$, respectively, in vitro. The presence of mono-n-butyl phthalate (MBP) and mono-2-ethylhexyl phthalate (MEHP) in saliva collected after chewing ball A was confirmed by GCMSSIM. Human saliva which collected from volunteers incubated with DBP and DEHP at $37^{\circ} \mathrm{C}$ over $60 \mathrm{~min}$, hydrolyzed these compounds to their monoesters.

Key words — dialkyl phthalate, monoester, hydrolysis, polyvinyle chloride toy, migration

\section{INTRODUCTION}

Dialkyl phthalates (PAEs) are widely used as plasticizers to impart softness and flexibility to normally rigid plastics such as polyvinyl chloride (PVC). Medical devices and toys are often made of PVC, containing the predominant plasticizers, di-2ethylhexyl phthalate (DEHP) or diisononyl phthalate (DINP). ${ }^{1,2)}$ Some PAEs have induced testicular toxicity and other effects on the male and female

*To whom correspondence should be addressed: Tokyo Kenbikyo-in Foundation, Center of Food \& Environmental Sciences, 44-1 Nihonbashi Hakozaki-cho, Chuo-ku, Tokyo 1030015, Japan. Tel.: +81-3-3663-9684; Fax: +81-3-3663-9682; Email: tatsu-n@jc4.so-net.ne.jp reproductive tract at high dosages in rats and other animals. ${ }^{3,4)}$ Jobling et al. showed that di- $n$-butyl phthalate (DBP) binds to the estrogen receptor, displaces the natural ligand from the receptor, then acts as an estrogen agonist rather than an antagonist. ${ }^{5)}$ The DEHP and DINP consist of many structurally dissimilar compounds known as peroxisome proliferators that induce tumors in the rodent liver. ${ }^{6}$

Orally-ingested PAE is hydrolyzed to its monoester by endogenous esterases of many tissues, such as the small intestine. ${ }^{7,8}$ Monoesters are thought to be more hepatocarcinogenic than their parent compound. ${ }^{9)}$

The rate of DINP release from PVC toys chewed by children has been measured using two general migration tests. An in vivo test in which human volunteers chew PVC toys under controlled conditions was developed by Meuling and Rijk in the Netherlands ${ }^{10)}$ and by Chen for the U.S. Consumer Product Safety Commission (USCPSC). ${ }^{11)}$ In addition, the effect of shaking or impacting PVC toys in simulated saliva in vitro has been tested. The release rates in vivo are higher than those obtained in vitro. However, the in vivo and in vitro migration of PAE has not been simultaneously studied except for DINP. Therefore, we examined the in vivo and in vitro migration of PAE from PVC toys containing DBP, DEHP and DINP. In addition, we examined monoester formation by the hydrolysis of PAE that migrated into saliva from PVC toys.

\section{MATERIALS AND METHODS}

Reagents — DBP and DINP were purchased from Kanto Chemical Co., Inc. (Tokyo, Japan) and DEHP from Wako Pure Chemical Ind. (Osaka, Japan). The purity of DBP, DEHP and DINP was over 99.7, 99.5 
and $97.9 \%$, respectively. Mono- $n$-butyl phthalate (MBP) and mono-2-ethylhexyl phthalate (MEHP) of over $90 \%$ purity were purchased from Tokyo Kasei Kogyo Co., Ltd. (Tokyo, Japan). Trimethylsilyldiazomethane (TMSD) was purchased from Aldrich Chemical Co. (U.S.A.). All other solvents and reagents were of analytical grade, and confirmed as being PAE free. The composition of the simulated saliva according to the British Standard Specification for Safety Harnesses was: $4.5 \mathrm{~g}$ sodium chloride, $0.3 \mathrm{~g}$ potassium chloride, $0.3 \mathrm{~g}$ sodium sulfate, $0.4 \mathrm{~g}$ ammonium chloride, $0.2 \mathrm{~g}$ urea and $3.0 \mathrm{~g}$ lactic acid dissolved in $1000 \mathrm{ml}$ distilled water adjusted to $\mathrm{pH} 6.5$ to 7.0 with $5 \mathrm{M}$ sodium hydroxide. ${ }^{12)}$ PAE and monoesters were solid-phase extracted using Oasis hydrophilic lipophilic balance (HLB) cartridges $(3 \mathrm{cc}$ ) from Waters Co. (Milford, Mass., U.S.A.). Prior to use, their cartridges were washed with $10 \mathrm{ml}$ of methanol, followed by $10 \mathrm{ml}$ of methanol $\bullet 0.1 \%$ acetate $(1: 9)$. Glassware was heated at temperatures over $230^{\circ} \mathrm{C}$ for at least $5 \mathrm{hr}$ before use.

HPLC Analysis — The HPLC conditions were as follows: apparatus, LC-10A (Shimadzu, Kyoto, Japan); column, Inertsil C8-3 (0.46 mm $\phi \times 250 \mathrm{~mm}$ ); column oven temperature, $40^{\circ} \mathrm{C}$; mobile phase, (A) water/acetic acid $200: 2(\mathrm{w} / \mathrm{w})$, (B) acetonitrile, A : B $=3: 7$; detector, UV (254 nm); injection volume, $10 \mu \mathrm{l}$.

GC/MS Analysis — The gas chromatography (GC) conditions were as follows: column, CBP5 $(0.25 \mathrm{~mm}$ i.d. $\times 50 \mathrm{~m}$, film thickness $0.25 \mu \mathrm{m})$; carrier gas, helium at $2.0 \mathrm{ml} / \mathrm{min}$; column temperature, $90^{\circ} \mathrm{C}(1 \mathrm{~min}) \rightarrow\left(40^{\circ} \mathrm{C} / \mathrm{min} \rightarrow 290^{\circ} \mathrm{C}\right.$; injection port and interface temperature, $290^{\circ} \mathrm{C}$. The MS conditions for electron impact ionization of monoalkyl phthalate were as follows: ion energy, $70 \mathrm{eV}$; ion source temperature, $270^{\circ} \mathrm{C}$; selected ion, $\mathrm{m} / \mathrm{z} 163$ and 149.

PAE Migration Test from PVC Toys — The PVC toy products were ball A that contained $100 \mathrm{mg} /$ $\mathrm{g}$ DBP and $190 \mathrm{mg} / \mathrm{g}$ DEHP, and ball B that contained $257 \mathrm{mg} / \mathrm{g}$ DINP. The balls were punched over a $2.5 \times 3.0 \mathrm{~cm}$ area (total surface area was approximately $15 \mathrm{~cm}^{2}$ ). These are referred to below as toy samples A or B.

Migration test in vitro: Rotary shaking migration was examined using a rotary shaker, VR-36 [TAITEC Co. (Saitama, Japan)]. Toy samples were placed in $50 \mathrm{ml}$ glass centrifuge tubes containing $30 \mathrm{ml}$ of simulated saliva. Tubes were placed on the rotary shaker in a incubator at $35^{\circ} \mathrm{C}$, and shaken for
$15 \mathrm{~min}$ at a rate of $300 \mathrm{rpm}$. The solutions $(5 \mathrm{ml})$ were sampled, and the rest was discarded. Four consecutive 15 min periods of this operation was repeated. The collected samples were mixed with $5 \mathrm{ml}$ of acetonitrile. Migrated PAE in the samples was quantified by HPLC.

Migration test in vivo: One female and three male volunteers gently chewed toy samples or control polypropylene (PP) disks PAE-free for $15 \mathrm{~min}$. They repeated this process for four $15 \mathrm{~min}$ intervals on toy sample A (four sessions), and were a 5 min break before actively sucking the sample. During each 15 min period, all produced saliva was collected in labeled $50 \mathrm{ml}$ glass centrifuge tubes. ${ }^{10,11)}$ The total volume and $\mathrm{pH}$ of produced saliva in each tube were recorded. All saliva samples were diluted to $10 \mathrm{ml}$ with distilled water, mixed with $10 \mathrm{ml}$ of acetonitrile, then sedimented by centrifugation at $3000 \mathrm{rpm}$ for $10 \mathrm{~min}$. This procedure was repeated by the same volunteers on toy sample B 1 week later. Migrated DBP and DEHP in the supernatant was quantified by HPLC.

The amount of monoalkyl phthalate in saliva samples was measured as follows. The supernatant was mixed with $15 \%$ sodium chloride and rendered alkaline with $0.1 \mathrm{~N}$ sodium hydroxide, then PAE was extracted with $n$-Hexane. Monoesters in the aqueous acetonitrile layer acidified with $0.01 \mathrm{~N}$ hydrochloric acid were extracted with $20 \%$ dichloromethane $-n$-hexane. The extracted monoesters were methyl-esterified with TMSD as described by Ohfuji et al. ${ }^{13)}$ Methyl-esterified monoesters were quantified by GC/MS.

\section{Incubation Test of PAE with Human Saliva}

Saliva $(0.5 \mathrm{ml})$ collected from volunteers who chewed PP disks was added to the same volume of simulated saliva and incubated with $50 \mathrm{nmol}$ of DBP or DEHP in $10 \mu \mathrm{l}$ of dimethylsulfoxide at $37^{\circ} \mathrm{C}$ for $5,15,30$ or $60 \mathrm{~min}$, respectively. The incubated mixture was adjusted to $\mathrm{pH} 4$ with $0.01 \mathrm{~N}$ hydrochloric acid, mixed with acetonitrile $(1 \mathrm{ml})$, then centrifuged at $3000 \mathrm{rpm}$ for $10 \mathrm{~min}$. The supernatants were mixed with $8 \mathrm{ml}$ of $0.1 \%$ acetic acid and passed through Oasis HLB cartridges. Organic compounds remaining in the column were eluted with $20 \mathrm{ml}$ of acetonitrile. The eluates were evaporated to near dryness, then PAE and monoesters were analyzed by HPLC. 
Table 1. Migration Rates of Dialkyl Phthalate (PAE) from PVC Toy Samples

\begin{tabular}{llcccr}
\hline \hline \multirow{2}{*}{ Toy sample } & PAE & $\begin{array}{c}\text { Contents } a) \\
(\mathrm{mg} / \mathrm{g})\end{array}$ & Migrated PAE $\left(\mu \mathrm{g} / 10 \mathrm{~cm}^{2} / \mathrm{hr}^{a}\right)$ & B \\
\cline { 4 - 5 } & & 100 & $11.7 \pm 9.8$ & $339 \pm 6.9$ & 28.9 \\
\hline Toy ball A & DBP & 185 & $44.4 \pm 12.3$ & $315 \pm 25.0$ & 6.6 \\
& DEHP & 256 & $78.0 \pm 28.9$ & $535 \pm 37.7$ & 6.9 \\
\hline
\end{tabular}

a) PAE contents in toy sample were measured by extraction with acetone using rotaty shaker at $300 \mathrm{rpm}$ for $3 \mathrm{hr}$. $b$ ) These release rates were obtained by in vivo migration tests after 4 volunteers chewed toy sample for $60 \min (15 \min \times 4$ sessions). Values are means \pm S.D. $(n=4)$. c $)$ These release rates were obtained by in vitro migration tests of simulated saliva shaken at $300 \mathrm{rpm}$ at $35^{\circ} \mathrm{C}$ for $60 \mathrm{~min}(15 \mathrm{~min} \times 4 \mathrm{sessions})$. Values are means \pm S.D. $(n=5)$.

\section{RESULTS AND DISCUSSION}

\section{In Vitro and In Vivo Migration of PAE}

Table 1 shows the release rates of PAE into human saliva (in vivo) and into simulated saliva (in vitro) from toy samples. The rates of DBP and DEHP migration into the simulated saliva were 339 and $315 \mu \mathrm{g} / 10 \mathrm{~cm}^{2} / \mathrm{hr}$, respectively $\left(654 \mu \mathrm{g} / 10 \mathrm{~cm}^{2} / \mathrm{hr}\right.$ at the sum total PAEs), and 11.7 and $44.4 \mu \mathrm{g} / 10 \mathrm{~cm}^{2} /$ hr into the human saliva, respectively $(56.1 \mu \mathrm{g} /$ $10 \mathrm{~cm}^{2} / \mathrm{hr}$ at the sum total PAEs). Those of DINP that migrated into the simulated and human saliva were $535 \mu \mathrm{g} / 10 \mathrm{~cm}^{2} / \mathrm{hr}$ and $78.0 \mu \mathrm{g} / 10 \mathrm{~cm}^{2} / \mathrm{hr}$, respectively. The release rates into saliva simulant were of the order DBP > DEHP > DINP. Although migration into saliva in vivo seemed to increase with shorter alkyl chains, about $70 \%$ less DBP and DEHP than DINP migrated. The amounts of DBP, DEHP and DINP that migrated in vivo were 28.9, 6.6 and 6.9- fold on the ratio of in vitro migration. Very little DBP migrated into saliva than DEHP and DINP.

The migration of DINP from PVC products in vivo has been tested in the Netherlands ${ }^{10)}$ and in the U.S.A. ${ }^{11)}$ The Dutch consensus study indicated a mean DINP release rate of $108 \mu \mathrm{g} / 10 \mathrm{~cm}^{2} / \mathrm{hr}$ from samples containing $430 \mathrm{mg} / \mathrm{g}$ of DINP which was considerably less than the American CPSC values of $219 \mu \mathrm{g} / 10 \mathrm{~cm}^{2} / \mathrm{hr}$. Generally, release rate and DINP content of the sample did not correlate. ${ }^{14)}$ Less DINP migrated from toy sample B than that indicated by the Dutch consensus study. Rates of saliva produced by chewing toy samples A and B were 37$92 \mathrm{ml} / \mathrm{hr}$ and $38-45 \mathrm{ml} / \mathrm{hr}$, and $\mathrm{pH}$ of the saliva was 7.0-7.3 and 6.7-7.6.

MBP and MEHP in saliva collected during the in vivo test of the toy sample A were methyl-esterified with TMSD, and analyzed by GC/MS. The methyl-esterified monoesters have a stable base peak at $m / z 163$ and 149 when analyzed by electron-impact MS. The chemical structure of these ions is shown in Fig. 1. Selective monitoring ions were used to identify monoesters in saliva extracts. The SIM chromatograms of the extracted saliva in Fig. 2 show the presence of MBP and MEHP indicating their formation through PAE conversion in saliva produced while chewing the toy sample.

\section{Hydrolysis of DBP and DEHP by Saliva to Form}

We examined the recovery of PAE and the monoester during the incubation test. Standard DBP, DEHP, MBP and MEHP added to simulated saliva

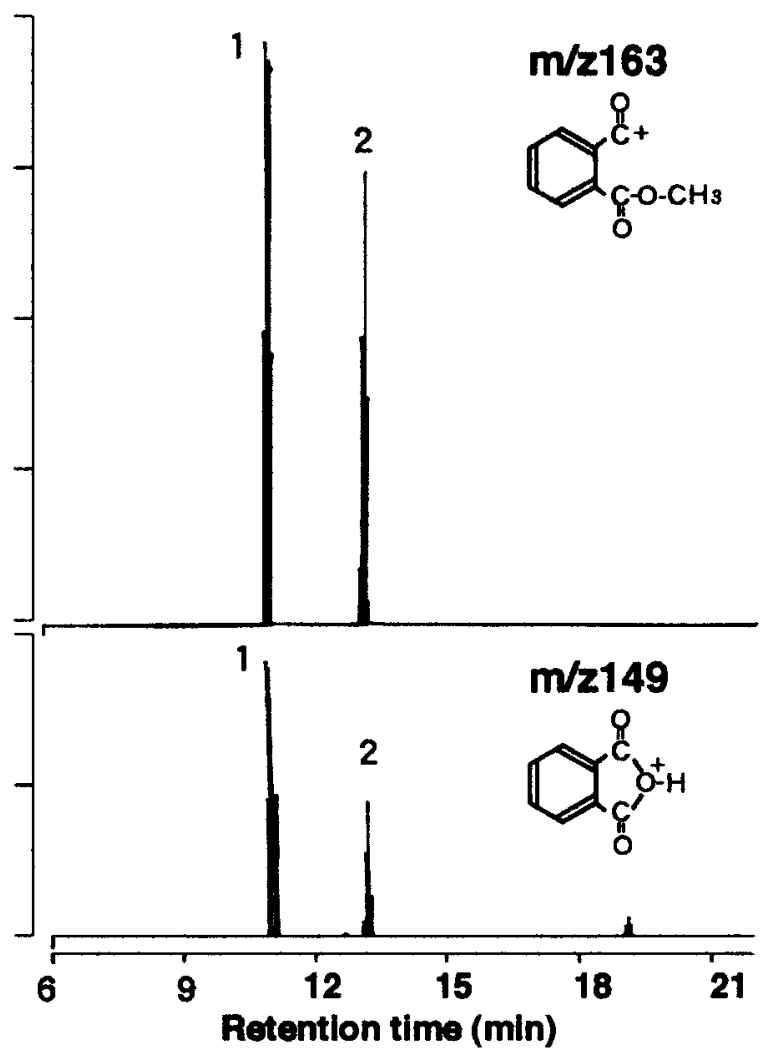

Fig. 1. GC/MS-SIM Trace of Methyl Esterified MBP and MEHP

1, mono- $n$-buthyl phthalate (MBP); 2, mono-2-ethylhexyl phthalate (MEHP). Amount of injected MBP and MEHP after methyl-esterification were $100 \mathrm{ng}$ each. 


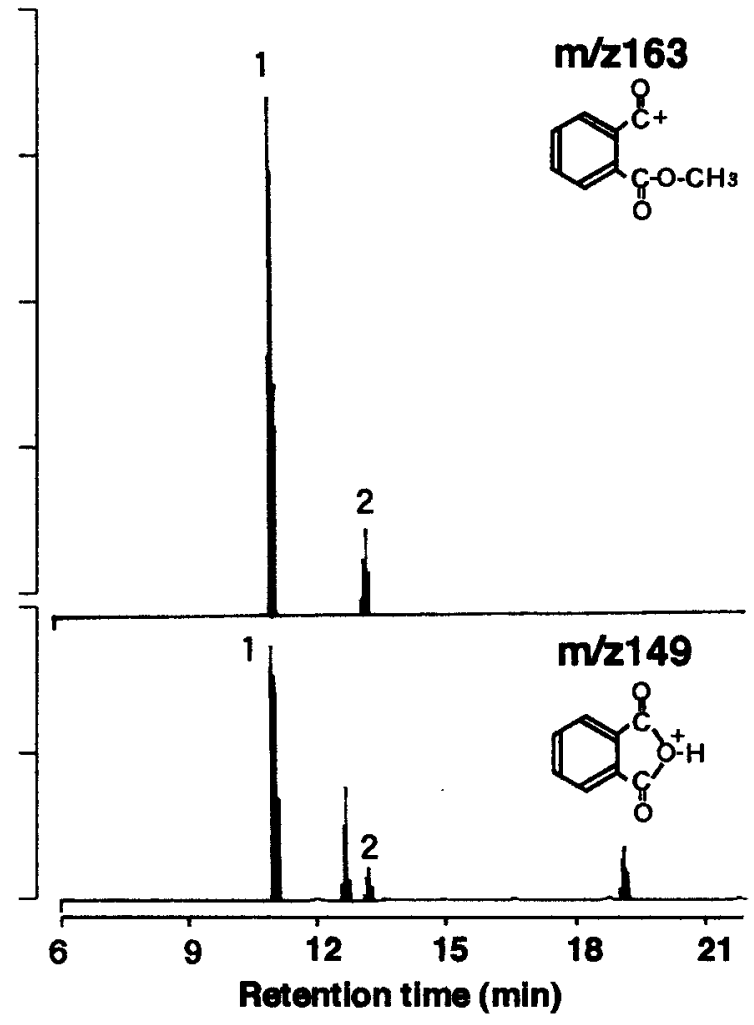

Fig. 2. GC/MS-SIM Trace of Methyl Esterified Saliva Extract Saliva was alkalified with $\mathrm{NaOH}$ and extracted with $n$-hexane. The lower layer was then acidified with HCI and extracted with $20 \%$ dichloromethane $\bullet n$-hexane.

were removed at rates of $96.8 \%, 83.1 \%, 99.4 \%$ and 99.6\% for DBP, DEHP, MBP, MEHP, respectively.

Figures 3 and 4 show the formation of MBP and MEHP by of saliva containing $50 \mathrm{nmol}$ of standard DBP and DEHP. The amounts of DBP and DEHP in the saliva decreased to $95 \%$ and $12 \%$, respectively over $60 \mathrm{~min}$ at $37^{\circ} \mathrm{C}$. MBP was formed from the added DBP within $5 \mathrm{~min}$. The MBP formation accounted for $87 \%$ of the added DBP within 60 min. On the other hand, the amount of MEHP slightly increased over $60 \mathrm{~min}$.

In general, PAE is thought to be metabolized to monoesters by esterases in the wall of the small intestine and by pancreatic lipases. ${ }^{78}$ DEHP is rapidly absorbed from the rodent gut, mostly in the form of the monoester because of the rapid hydrolysis of the DEHP by gut lipases. ${ }^{15,16)}$ The present studies showed that MBP and MEHP were formed by the monohydrolysis of DBP and DEHP in human saliva. We surmised that the hydrolysis of PAE in human saliva differs according to the length of the alkyl chains, because more MBP was formed from DBP than MEHP from DEHP. Kayano et al. reported that the hydrolysis of PAE by the mouse hepatic esterase

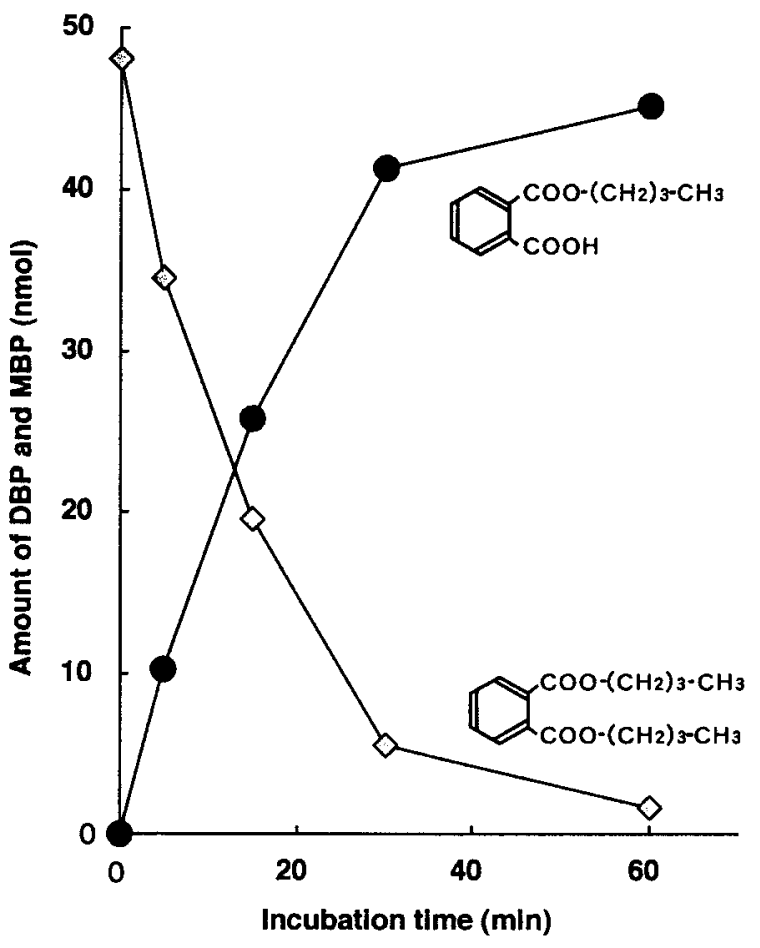

Fig. 3. The Relationship between MBP Formation by DBP Hydrolysis and Incubation Time in the Human Saliva

Saliva obtained from volunteers who had chewed PP disk was mixed with $50 \mathrm{nmol} \mathrm{DBP}$ and incubated at $37^{\circ} \mathrm{C}$ for $60 \mathrm{~min}$. Values are means of diplicate assays. $\diamond$, Amount of DBP. $\bullet$, Amount of MBP.

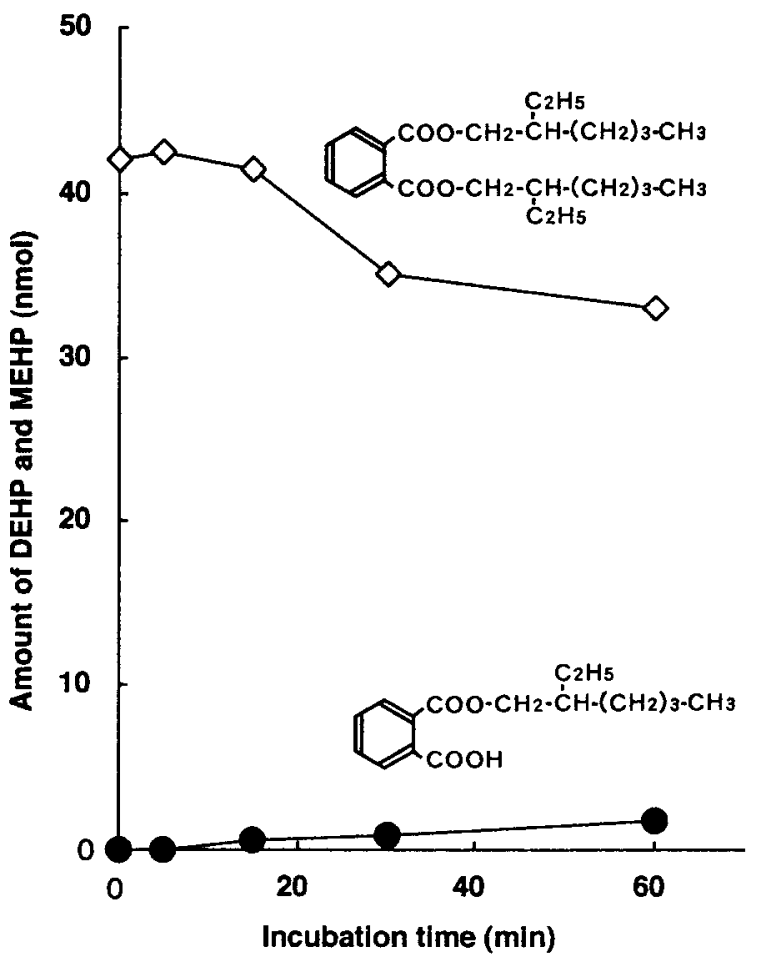

Fig. 4. The Relationship between MEHP Formation by DEHP Hydrolysis and Incubation Time in the Human Saliva Saliva obtained from volunteers who had chewed PP disks was mixed with $50 \mathrm{nmol}$ DEHP and incubated at $37^{\circ} \mathrm{C}$ for $60 \mathrm{~min}$. Values are means of diplicate assays. $\diamond$, Amount of DEHP. $\bullet$, Amount of MEHP. 
has substrate specificity for chained alkyl groups. ${ }^{17)}$ The MEHP compounds are thought to be more hepatocarcinogenic and teratogenic than DEHP at high dosages. ${ }^{9}$ In conclusion, the results of the present study indicated that infants and toddlers can be exposed to PAE by chewing PVC toys, and that the monoester may be formed through hydrolysis by saliva in the oral cavity.

Acknowledgements This study was supported by the "Health Science Research Grants, 1999" from the Ministry of Health and Welfare of Japan.

\section{REFERENCES}

1) Barry, Y. A., Labow, R. S., Keon, W. J., Tocchi, M. and Rock, G. (1989) Perioperative exposure to plasticizers in patients undergoing cardiopulmonary bypass. J. Thorac. Cardiovasc. Surg., 97, 900-905.

2) Bartlett, S. (1998a) Risk assessment on diisononyl phthalate in vinyl children's products. Investigation Report, Consumer Products Division, Product Safety Bureau, Environmental Health Directorate, Health Protection Branch, Health Canada, Ottawa, Canada.

3) Gray, T. J. B. and Gangolli, S. D. (1986) Aspects of the testicular toxicity of phthalate esters. Environ. Health Perspect., 65, 229-235.

4) Wine, R. N., Li, L.-H., Barnes, L. H., Gulati, D. K. and Chapin, R. E. (1997) Reproductive toxicity of di- $n$-butylphthalate in a continuous breeding protocol in Sprague-Dawley rats. Environ. Health Perspect., 105, 102-107.

5) Jobling, S., Reynolds, T., White, R., Parker, M. G. and Sumpter, J. P. (1995) A Variety of environmentally persistent chemicals, including some phthalate plasticizers, are weakly estrogenic. Environ. Health Perspect., 103, 582-587.

6) International Agency for Research on Cancer (1995) Peroxisome Proliferation and its Role in Carcinogenesis, International Agency for Research on Cancer (IARC), World Health Organization, Lyon. IARC Technical Report No. 24.

7) Lake, B. G., Phillips, J. C., Linnell, J. C. and Gangolli, S. D. (1977) The in vitro hydrolysis of some phthalate diesters by hepatic and intestinal preparations from various species. Toxicol. Appl. Pharmacol., 39, 239-248.

8) Rowland, I. R., Cottrell, R. C. and Phillips, J. C.
(1977) Hydrolysis of phthalate esters by the gastrointestinal contents of the rat. Food Cosmet. Toxicol., 15, 17-21.

9) Nakamura, Y. and Tomita, I. (1987) Futarusanesuteru no genjyo to mondaiten. Jpn. J. Toxicol. Environ. Health, 33, 71-89.

10) RIVM, Milieu RVVE (1998) Phthalate release from soft PVC baby toys. RIVM report 613320 002: National Institute of Public Health and the Environment.

11) Chen, S. B. (1998b) Migration of DINP from polyvinyl chloride (PVC) children's products. In The risk of chronic toxicity associated with exposure to diisononyl phthalate (DINP) in children's products (Babich, A. M., Ed.), U.S. Consumer Product Safety Commission, Washington, D.C.

12) British Standard Specification for safety harnesses (including detachable walking reins) for restraining children when in perambulators (baby carriages), pushchairs and high chairs and when walking, BS6684: 1987.

13) Ohfugi, M., Chikamoto, T., Kamada, I. and Komatsu, M. (1997) A Gas chromatographic method for determination of dinoseb, dinoterb and 4,6dinitro-o-cresol (DNOC) in citrus fruits by methylation using trimethylsilyldiazomethane. Shokuhin Eiseigaku Zasshi (J. Food Hyg. Soc. Japan), 38, 85-91.

14) Health Canada (1998b) Study on Phthalates in Children's Plastic Consumer Products, Project No. 97-0428A., Product Safety Laboratory, Product Safety Bureau, Environmental Health Directorate, Health Protection Branch.

15) Eriksson, P. and Darnerud, P. (1986) Distribution and retention of some chlorinated hydrocarbons and a phthalate in the mouse brain during the preweaning period. Toxicology., 37, 189-204.

16) Pollack, G. M., Li, R. C. K., Ermer, J. C. and Shen, D. D. (1985) Effects of route of administration and repetitive dosing on the disposition kinetics of di (2-ethylhexyl) phthalate and its mono-de-esterfied metabolite in rats. Toxicol. Appl. Pharmacol., 79, 246-256.

17) Kayano, Y., Watanabe, K., Matsunaga, T., Yamamoto, I. and Yoshimura, H. (1995) Involvement of a novel mouse hepatic esterase (ES46.5K) in the hydrolysis of phthalate esters. "Abstr. 21st Symp. Toxicol. Env. Health," Osaka, pp. 51-52. 